\title{
NOTE ON CLOSURE OF ORTHOGONAL SETS.
}

\author{
BY PROFESSOR O. D. KELLOGG.
}

(Read before the American Mathematical Society April 24, 1920.)

\section{Introduction.}

The present note has to do with the set $\left[\varphi_{i}(x)\right](i=0,1,2$, ...) of solutions of a differential equation

$$
\frac{d}{d x}\left(k \varphi^{\prime}\right)+(\lambda g-l) \varphi=0,
$$

in which $k, g$, and $l$ are continuous, and $k>0(a \leqq x \leqq b)$, $g>0(a<x<b)$, the solutions satisfying a pair of homogeneous linear self-adjoint boundary conditions

$$
\begin{aligned}
& U_{1}(\varphi)=a_{1} \varphi(a)+a_{2} \varphi^{\prime}(a)-a_{3} \varphi(b)-a_{4} \varphi^{\prime}(b)=0, \\
& U_{2}(\varphi)=b_{1} \varphi(a)+b_{2} \varphi^{\prime}(a)-b_{3} \varphi(b)-b_{4} \varphi^{\prime}(b)=0,
\end{aligned}
$$

the condition for self-adjointness being

$$
k(a)\left(a_{3} b_{4}-a_{4} b_{3}\right)=k(b)\left(a_{1} b_{2}-a_{2} b_{1}\right) .
$$

Such a set is called closed with respect to functions of a given class, provided there is no function of the class orthogonal to all the functions of the set, i.e., no function $f$ such that $\int_{a}^{b} f \varphi_{i} g d x=0,(i=0,1,2, \cdots)$. Closure of a set is evidently implied whenever it is known that a function of a given class is uniquely determined by its generalized Fourier constants, and in this way a large number of closure theorems are at hand. Stekloff* has proved that for the case of the Sturm-Liouville boundary conditions, in which $a_{3}=a_{4}=b_{1}=b_{2}$ $=0$, the set of solutions is closed with respect to the class of functions that are integrable with integrable squares. Hilbert, $\dagger$ using boundary conditions in part special cases of (2) above, and in part going beyond, in that singularities of the differential

* Annales de la Faculté des Sciences de Toulouse, 2d ser., vol. 3 (1901); Mémoires de l'Académie Impériale des Sciences de St.-Petersbourg, vol. 30, No. 4 (1911).

† Göttinger Nachrichten, 1904, p. 222. 
equation at the end points of the interval $(a, b)$ are considered, shows that the sets studied are closed with respect to the class of continuous functions. In the following, I wish first to call attention to an easily derived identity (7) from which it may be inferred immediately that if the set of solutions of the differential system (1) and (2) is closed with respect to the class $C$ of continuous functions not zero, it is then also closed with respect to the class $S$ of summable* functions not null functions. A second paragraph will indicate an application of the method of successive approximations from which the existence of the set of solutions may be inferred, as well as its closure with respect to functions of class $S$.

\section{The Relative Closure Identity.}

Let $f(x)$ be a function of class $S$, and let $\mu$ be a value of $\lambda$ for which the system (1) and (2) has no solution except 0 . Then the differential equation

$$
\frac{d}{d x}\left(k w^{\prime}\right)+(\mu g-l) w=f g
$$

has a solution in the following sense: there exists a function $w(x)$, continuous, together with its first derivative, which satisfies the boundary conditions (2), and which satisfies (4) except at most at points of a set of measure 0 . To see this, take two solutions, $v_{1}(x)$ and $v_{2}(x)$, of the homogeneous equation obtained from (4) by replacing the right-hand member by 0 ; these may be chosen so that $k(x)\left(v_{1} v_{2}{ }^{\prime}-v_{1}{ }^{\prime} v_{2}\right) \equiv 1$. Then

$$
w_{0}(x)=\int_{a}^{x}\left|\begin{array}{ll}
v_{1}(\xi), & v_{2}(\xi) \\
v_{1}(x), & v_{2}(x)
\end{array}\right| f(\xi) g(\xi) d \xi
$$

is continuous, since the indefinite integral of a summable function is continuous. The same is true of its derivative. Finally, $k w^{\prime}$, since the indefinite integral of a summable function has a derivative equal to the integrand, except at most at points of a set of zero measure (Lebesgue, l. c., page 124-125), has a derivative except at points of a null set. It is immediately seen that in this sense $w_{0}(x)$ is a particular solution of (4).

* In the sense of Lebesgue. See Leçons sur l'Intégration et la Recherche des Fonctions primitives, p. 115. 
From $w_{0}(x)$ one forms the solution which satisfies (2):

(6) $w(x)=\left|\begin{array}{lll}w_{0}(x) & v_{1}(x) & v_{2}(x) \\ U_{1}\left(w_{0}\right) & U_{1}\left(v_{1}\right) & U_{1}\left(v_{2}\right) \\ U_{2}\left(w_{0}\right) & U_{2}\left(v_{1}\right) & U_{2}\left(v_{2}\right)\end{array}\right| \div\left|\begin{array}{ll}U_{1}\left(v_{1}\right) & U_{1}\left(v_{2}\right) \\ U_{2}\left(v_{1}\right) & U_{2}\left(v_{2}\right)\end{array}\right|$,

where the denominator determinant does not vanish, since $\mu$ was a parameter value for which the homogeneous problem was not possible. Let now $\varphi_{i}(x)$ be any one of the set of solutions of (1) and (2), and let $\lambda_{i}$ be the corresponding value of $\lambda$. From (1) and (4) by a familiar process, we get

$$
\frac{d}{d x} k\left(\varphi_{i} w^{\prime}-\varphi_{i}^{\prime} w\right)+\left(\mu-\lambda_{i}\right) w \varphi_{i} g=f \varphi_{i} g .
$$

This equation, failing at most on a set of measure 0 , becomes an identity upon integration between the limits $a$ and $b$. Since $\varphi_{i}$ and $w$ satisfy (2), which are self-adjoint, and (3), the integrated terms disappear, and we are led to

$$
\left(\mu-\lambda_{i}\right) \int_{a}^{b} w \varphi_{i} g d x=\int_{a}^{b} f \varphi_{i} g d x,
$$

the identity referred to in the introduction. Since $f$ is not identically zero, $w$ cannot be, by (4), and so is of class $C$. The identity shows that $w$ is orthogonal to every function $\varphi_{i}$ to which $f$ is orthogonal and the theorem stated follows.

\section{Existence and Closure of the Set $\left[\varphi_{i}(x)\right]$.}

We start with the functions $f$ and $w$ above, without, however, supposing anything about their orthogonality to functions of the set $\left[\varphi_{i}(x)\right]$. We define a sequence $w_{1}, w_{2}, w_{3}, \cdots$ as follows:

$$
\begin{aligned}
w_{i 0} & =\int_{a}^{x}\left|\begin{array}{ll}
v_{1}(\xi), & v_{2}(\xi) \\
v_{1}(x), & v_{2}(x)
\end{array}\right| \bar{w}_{i-1}(\xi) g(\xi) d \xi, \\
\text { (8) } w_{i} & =\left|\begin{array}{lll}
w_{i 0}(x) & v_{1}(x) & v_{2}(x) \\
U_{1}\left(w_{i 0}\right) & U_{1}\left(v_{1}\right) & U_{1}\left(v_{2}\right) \\
U_{2}\left(w_{i 0}\right) & U_{2}\left(v_{1}\right) & U_{2}\left(v_{2}\right)
\end{array}\right| \div\left|\begin{array}{ll}
U_{1}\left(v_{1}\right) & U_{1}\left(v_{2}\right) \\
U_{2}\left(v_{1}\right) & U_{2}\left(v_{2}\right)
\end{array}\right|, \\
\bar{w}_{i} & =w_{i} / \sqrt{\int_{a}^{b} w_{i}^{2} g d x} \\
w_{1} & =w .
\end{aligned}
$$


These functions satisfy at all points the differential equations

$$
\frac{d}{d x}\left(k w_{i}{ }^{\prime}\right)+(\mu g-l) w_{i}=\bar{w}_{i-1} g .
$$

We proceed to consider the existence and properties of limit functions of the sequence $\left[w_{i}\right]$. We first note that $w_{i}$ and $w_{i}{ }^{\prime}$ are bounded by a number $B$ independent of $i$. For, if $M$ is greater than the maxima of $\left|v_{1}\right|,\left|v_{1}{ }^{\prime}\right|,\left|v_{2}\right|,\left|v_{2}{ }^{\prime}\right|$, and $g$, we have by Schwarz's inequality applied to $\left(8_{1}\right),\left|w_{i}\right|$ and $\left|\begin{array}{c}w_{i}{ }^{\prime} \\ w_{i}{ }^{\prime}\end{array}\right|<2 M \sqrt{M(b-a)}$, so that a bound, $B$, for $\left|w_{i}\right|$ and

Next, writing the equation (9) and the same equation with $i+1$ replacing $i$, multiplying these equations respectively by $\bar{w}_{i+1}$ and $\bar{w}_{i}$, subtracting, and integrating from $a$ to $b$, we find $\int_{a}^{b} w_{i+1} \bar{w}_{i-1} g d x=\int_{a}^{b} w_{i} \bar{w}_{i} g d x$. If in this equation we replace the value of the normed functions by their values in terms of the un-normed functions, we find

$$
\int_{a}^{b} w_{i+1} w_{i-1} g d x=\sqrt{\int_{a}^{b} w_{i}{ }^{2} g d x \cdot \int_{a}^{b} w_{i-1}^{2} g d x}
$$

From this we infer two things. First, by Schwarz's inequality, that $\int_{a}^{b} w_{i}{ }^{2} g d x \leqq \int_{a}^{b} w_{i+1}{ }^{2} g d x$, and hence, that the bounded numbers $\int_{a}^{b} w_{i}^{2} g d x$ approach a limit $c^{2}$, and secondly, that the integral $\int_{a}^{b} w_{i} w_{i+2} g d x$ also approaches $c^{2}$.

We now know that because of the boundedness of the derivatives of $w_{i}$, there exists a sub-sequence taken from the $w_{2 i}$ which approaches a limit $W_{0}{ }^{*}$ This limit is approached uniformly, and is continuous. If we denote by $\left[w_{2 i^{i}}\right]$ the subsequence approaching $W_{0}$ uniformly, then (8) shows that $w_{2^{\prime}+1}$ also approaches a limit $W_{1}$, and that this function satisfies the differential equation

$$
\frac{d}{d x}\left(k W_{1}^{\prime}\right)+(\mu g-l) W_{1}=W_{0} g / c .
$$

*Cf. Osgood, Annals of Mathematics, vol. 14 (1913), p. 182. 
Similarly $w_{2 i^{\prime}+2}$ approaches a limit, $W_{2}$, say. But this limit cannot differ from $W_{0}$, since

$$
\begin{aligned}
\int_{a}^{b}\left(w_{2 i^{\prime}}-w_{2 i^{\prime}+2}\right)^{2} g d x & =\int_{a}^{b} w_{2 i^{\prime}}^{2} g d x-2 \int_{a}^{b} w_{2 i^{\prime}} w_{2 i^{\prime}+2} g d x \\
+ & \int_{a}^{b} w_{2 i^{\prime}+2}^{2} g d x
\end{aligned}
$$

approaches zero, as we have just seen. Hence

$$
\int_{a}^{b}\left(W_{2}-W_{0}\right)^{2} g d x=0,
$$

and the continuous functions $W_{2}$ and $W_{0}$ are equal. We infer further that

$$
\frac{d}{d x}\left(k W_{0}^{\prime}\right)+(\mu g-l) W_{0}=W_{1} g / c .
$$

The norms of $W_{0}$ and $W_{1}$ are equal to $c$, so that either their sum or their difference is not identically zero. But $W_{0}+W_{1}$ is a solution of (1) and (2) corresponding to $\lambda=\mu-1 / c$ and $W_{0}-W_{1}$ is a solution corresponding to $\lambda=\mu+1 / c$.

Having one solution of the homogeneous problem, we need merely start with a function $f$ orthogonal to it in order to arrive by the above process at a new one. In this way may be inferred the existence of an infinite set of solutions. A simple procedure would be to start with a function $f(x)$ having a break in its derivative but continuous. As such a function cannot be a finite sum of functions $c_{i} \varphi_{i}(x)$, it may, after $\varphi_{1}, \varphi_{2}, \varphi_{3}, \cdots$ have been found, be made successively orthogonal to these by subtraction of proper multiples of them, without reducing to zero, and so yield, in time, any function of the set.

The set containing all the solutions of (1) and (2) is then closed with respect to the class $S$. For, if not, there would be a function $f$ of class $S$ orthogonal to all $\varphi_{i}$, and starting the process developed above with this $f$, we should ultimately arrive at the contradiction that $W_{0}+W_{1}$ or $W_{0}-W_{1}$, that is, one of the $\varphi_{i}$ itself, was orthogonal to the set.

CAMBRIDGe, Mass., August 23, 1920. 